Interactive comment on "Mitigation of the double ITCZ syndrome in BCC-CSM2-MR through improving parameterizations of boundary-layer turbulence and shallow convection" by Yixiong Lu et al.

\title{
David Ham
}

david.ham@imperial.ac.uk

Received and published: 10 July 2020

This is an executive editor comment highlighting the ways in which this manuscript is not currently compliant with GMD policy on code and data availability.

This manuscript currently indicates that code and data is available on request from the author. This does not comply with GMD policy on code and data availability, and the manuscript must be rejected unless this can be remedied. In particular, code and data must be archived and published at the time of submission in order to facilitate the open 
review process.

Please immediately archive and publish the code and data on which this manuscript depends and post the citations in a reply to this comment. Naturally, the code and data must be properly cited in the revised manuscript.

The full details of the code and data availability requirements are in the GMD model code and data policy: https://www.geoscientific-model-development.net/about/code_ and_data_policy.html. The reasons for the policy and more detail are provided in this editorial: https://doi.org/10.5194/gmd-12-2215-2019.

Interactive comment on Geosci. Model Dev. Discuss., https://doi.org/10.5194/gmd-2020-40, 2020. 